\title{
Registros de cetáceos del Mioceno Tardío de Entre Ríos, Argentina
}

\author{
Records of cetaceans from the Late Miocene from Entre Ríos, Argentina \\ Federico Agnolin ${ }^{1}$ y Sergio Lucero ${ }^{2}$ \\ ${ }^{1}$ Museo Argentino de Ciencias Naturales "Bernardino Rivadavia", Av. Angel Gallardo 470, (1405), Buenos Aires, Argentina \\ ${ }^{2}$ Facultad de Ciencias Naturales y Museo, Universidad Nacional de La Plata. Calles 60 y 122 (1900), La Plata, Argentina \\ fedeagnolin@yahoo.com.ar
}

\begin{abstract}
Records of cetaceans from the Late Miocene from Entre Rios, Argentina. In this paper cetacean remains from the Paraná Formation (Late Miocene) of the Entre Ríos province, Argentina are described. The following groups are described in detail from the Paraná Formation: Balaenidae, Balaenopteridae (Mysticeti), Platanistoidea and Physeteridae
\end{abstract}

\section{Introducción}

La Formación Paraná (Mioceno Tardío) localizada en la Provincia de Entre Ríos, Argentina, ha sido la primera unidad en brindar registros fósiles de mamíferos marinos para Sudamérica (Bravard, 1858). Durante mucho tiempo el registro de cetáceos fósiles para esta Formación ha estado restringido únicamente al odontoceto pontopórido Pontistes rectifrons (Cabrera 1926, de Muizon 1988). Posteriormente, Cozzuol (1996) menciona, sin ilustrar ni describir, las familias Balaenidae y Balaenopteridae (Mysticeti) así como Physeteridae (Odontoceti).

En este trabajo se describen por primera vez restos fósiles asignables a las familias Balaenidae, Balaenopteridae y Physeteridae procedentes de la Formación Paraná (Mioceno Tardío).

Para la identificación y nominación del material osteológico se utilizó la nomenclatura utilizada por Luo (1998) y Kasuya (1973). Para la sistemática de los cetáceos misticetos seguimos a Sanders \& Barnes (2002) y para la de los cetáceos odontocetos a de Muizon (1991).

\section{Abreviaturas}

MACN Pv, Colección de Paleontología de Vertebrados del Museo Argentino de Ciencias Naturales "Bernardino Rivadavia”.
(Odontoceti). The Balaenopteridae record represents the ancient for the family in Argentina.

Key words: Upper Miocene, cetaceans, Mysticeti, Odontoceti, marine mamals, paleontology, fossil records

\section{Paleontología sistemática}

\author{
Orden Cetacea Brisson, 1762 \\ Suborden Odontoceti Flower, 1867 \\ Familia Physeteridae Gray, 1821 \\ Subfamilia Haplocetinae sensu de \\ Muizon, 1991 \\ cf. Aulophyseter sp.
}

\section{Material referido}

MACN Pv 8926, diente incompleto (Fig. 1A).

\section{Descripción}

Diente de gran tamaño (la porción conservada de la corona exhibe una altura de $10 \mathrm{~cm}$ ). La corona carece de un cuello basal y se presenta levemente curvada hacia el margen posterior. Proximalmente, es de sección circular y distalmente, es de sección ovoidal. La corona presenta una amplia capa de esmalte rugoso, asemejándose a los extintos Haplocetinae (de Muizon, 1991). La corona no presenta una cresta lateral y aparentemente carece del cíngulo bien marcado característico de los Physeteridae primitivos (ej. Prealophyseter Caviglia \& Jorge 1980). El diente no presenta evidencias de un cuello basal como presenta el género Scaldicetus (Kellogg 1944, Morgan 1994). 


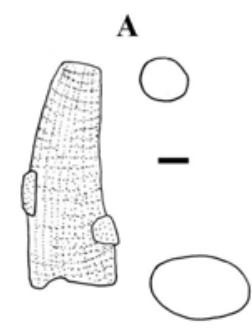

B

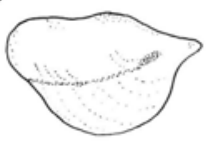

C

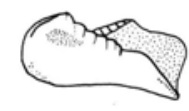

D

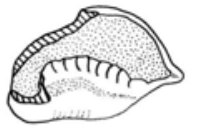

F
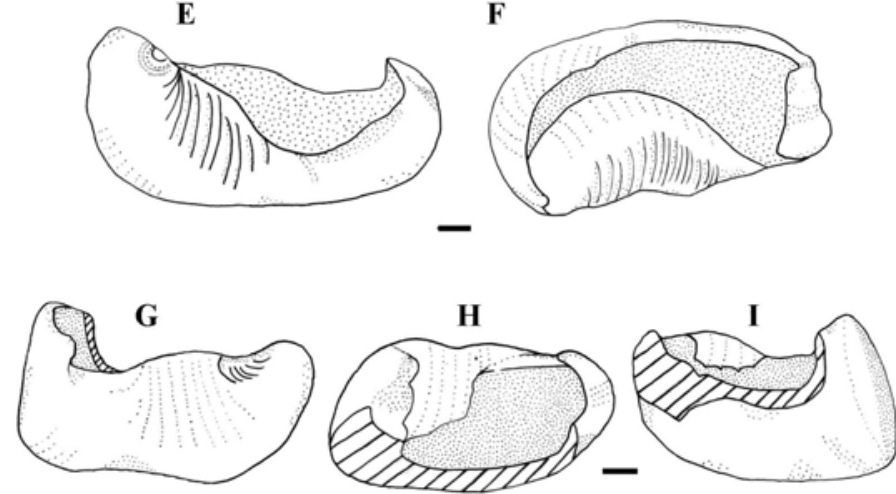

Figura 1

A, cf. Aulophyseter (MACN Pv 8926) diente incompleto en vista lateral y sección; B-D, Platanistoidea indet. (MACN Pv 15997) bulla timpánica izquierda en vistas ventral (B), lateral (C) y dorsal (D); E-F, Balaenoptera sp. (MACN Pv 13220) bulla timpánica derecha en vistas lateral (E), dorsal (F); G-I, Balaenidae indet. (MACN Pv 13221) bulla timpánica derecha en vistas lateral (G), dorsal (H), medial (I). Escala $1 \mathrm{~cm}$

A, cf. Aulophyseter (MACN Pv 8926) incomplete tooth in lateral view and cross section; B-D, Platanistoidea indet. (MACN Pv 15997) left tympanic bulla in ventral (B), lateral (C) and dorsal (D) views; E-F, Balaenoptera sp. (MACN Pv 13220) right tympanic bulla in lateral (E) and dorsal (F) views; G-I, Balaenidae indet. (MACN Pv 13221) right tympanic bulla in lateral $(\mathrm{G})$, dorsal $(\mathrm{H})$ and medial (I) views. Scale bar $1 \mathrm{~cm}$

\section{Discusión}

El diente aquí descripto es asignable a la subfamilia Haplocetinae por retener una amplia capa de esmalte en la corona, a diferencia de los Physeterinae, en los cuales esta capa se encuentra ausente (sensu de Muizon 1991). Adicionalmente, difiere de la mayoría de los miembros de esta última subfamilia porque éstos se caracterizan por presentar dientes menos comprimidos lateralmente (subcilíndricos en sección). Genéricamente, el diente descripto es semejante tanto en morfología (curvatura de la corona y rugosidad del esmalte) como en proporciones al extinto Aulophyseter (véase Gondar, 1975), sin embargo, el estado fragmentario del material impide una asignación genérica precisa.

Orden Cetacea Brisson, 1762

Suborden Odontoceti Flower, 1867

Superfamilia Platanistoidea Gray, 1863

Familia indeterminada

Género y especie indeterminados

\section{Material referido}

MACN Pv 15997, bulla timpánica izquierda (Fig. 1 BD).

\section{Descripción}

La bulla es baja y ancha, de contorno ovoidal. Presenta el involucro ancho y robusto, surcado por algunas estrías profundas. En vista lateral, el involucro es recto y disminuye anteriormente en tamaño. El canal de eustaquio es profundo y muy amplio, estrechándose anteriormente. El proceso anterior es agudo y presenta una terminación en forma cónica, bien desarrollada, pero menos protruyente que en la familia Platanistidae (Kasuya 1973). Ventralmente la bulla presenta el surco medio recto y profundo, el cual no llega hasta el extremo cranial del proceso anterior, a diferencia de lo que ocurre en los Platanistidae (Kasuya, 1973). El proceso posterior externo es estrecho y ventralmente liso. 


\section{Discusión}

La bulla aquí descripta es asignable a la superfamilia Platanistoidea por la siguiente combinación de caracteres: 1) bulla ensanchada lateralmente, 2) proceso posterior externo de pequeño tamaño, 3) involucro en vista lateral no sigmoideo (Kasuya 1973, de Muizon et al. 2002). Adicionalmente el material se asemeja a la familia Platanistidae en la existencia de una espina anterior y en que la pared lateral de la bulla es muy convexa (Kasuya 1973). Sin embargo, el material aquí descripto se distingue de los Platanistidae por presentar la pared lateral baja y el canal de eustaquio muy ancho. Debido a esta combinación de caracteres el material no puede ser asignado con certeza a ninguna familia conocida dentro de Platanistoidea.

Suborden Mysticeti Flower, 1864

Superfamilia Balaenopteroidea (Gray, 1868) Familia Balaenopteridae Gray, 1864 Subfamilia Balaenopterinae (Gray, 1864)

Género Balaenoptera Lacépède, 1804 Balaenoptera sp.

\section{Material referido}

MACN Pv 13220, bulla timpánica derecha (Fig. 1 E-F).

\section{Descripción}

La bulla es baja y elongada, de contorno oblongo, presenta el margen ventral fuertemente convexo. En vista dorsal la bulla se encuentra medianamente dilatada y presenta un ancho uniforme. El involucro es ancho, bajo y fuertemente estriado. El envolvimiento anterior del involucro es bastante delgado. El pedicelo posterior no se encuentra preservado, sin embargo su base indica que sería de pequeño tamaño. El canal de eustaquio es profundo y muy amplio, estrechándose en su margen posterior y delimitado por el envolvimiento del involucro en su margen anterior. La cara lateral de la bulla es cóncava. Ventralmente presenta el surco medial recto y poco profundo.

\section{Discusión}

El material aquí descripto es asignable al suborden Mysticeti, debido al fuerte estriado presente en el involucro de la bulla, y ha sido asignado a la familia Balaenopteridae por presentar la bulla de contorno oblongo (Gondar, 1966) y estrecha. Genéricamente es asignable a Balaenoptera por la siguiente combinación de caracteres: 1) bulla convexa en su cara ventral, 2) bulla medianamente dilatada y de ancho uniforme y 3) cara medial de la bulla cóncava. Debido a la condición fragmentaria del material es imposible ajustar una clasificación inferior a la genérica. No obstante, cabe señalar que la bulla es proporcionalmente mas baja que la de las especies conocidas de Balaenoptera.

La presencia de posibles Balaenopteridae en la Formación Paraná fue sugerida por Ameghino (1889), refiriéndose a la posición sistemática de la enigmática Balaena dubia (nomen nudum) de Bravard (1858). El material aquí descripto representaría el primer registro fehaciente para la familia Balaenopteridae en la Formación Paraná y constituiría el más antiguo para la familia antedicha en Argentina.

\section{Superfamilia Balaenoidea (Brandt, 1873) \\ Familia Balaenidae Gray, 1825 Género y especie indeterminados}

\section{Material referido}

MACN Pv 13221, bulla timpánica derecha (Fig. 1 G-I).

\section{Descripción}

La bulla es alta, corta y de contorno romboidal. El involucro es bajo y se encuentra levemente estriado. El envolvimiento anterior del involucro es relativamente grueso y muy elevado dorsalmente. Canal de eustaquio muy ancho, pero poco profundo. Ventralmente la bulla presenta un surco medial sigmoideo y poco profundo, característica que diferencia esta bulla de las pertenecientes a las familias Balaenopteridae y Cetotheriidae, en las cuales este surco es recto.

\section{Discusión}

La bulla aquí descripta puede ser incluida dentro de la familia Balaenidae por presentar el contorno general romboidal (Gondar 1966) y el surco ventro-medial sigmoideo y poco profundo. Sin embargo, la bulla timpánica aquí descripta es más baja y de tamaño mucho menor que la que presentan los géneros actuales Eubalaena y Balaena. Debido a las diferencias que esta bulla exhibe con los géneros existentes no puede ser asignada con seguridad a ningún taxón conocido.

\section{Conclusiones}

Restos asignables a las familias Physeteridae (subfamilia Haplocetinae), Balaenidae y Balaenopteridae (subfamilia Balaenopterinae) son descriptos en detalle por primera vez para la Formación Paraná. 
La familia Balaenopteridae se encuentra representada por restos asignables al género viviente Balaenoptera, constituyendo la mención mas antigua para la familia y el género en Argentina.

La familia Balaenidae se encuentra representada por restos pertenecientes probablemente a una nueva entidad genérica.

Se registra la existencia de un odontoceto indeterminado perteneciente a la superfamilia Platanistoidea, el cual posiblemente represente un nuevo taxón aún no reconocido.

Los cetáceos de la Formación Paraná se encuentran representados por una gran diversidad, contándose con al menos cinco géneros distribuidos en las familias Pontoporiidae, Physeteridae, Balaenidae y Balaenopteridae y un Platanistoidea indeterminado.

\section{Agradecimientos}

Agradecemos a A Kramarz y JF Bonaparte por pemitirnos el acceso a la colección de Paleontología de Vertebrados del Museo Argentino de Ciencias Naturales “Bernardino Rivadavia”. Finalmente agradecemos a dos revisores anónimos por la lectura crítica del manuscrito.

\section{Literatura citada}

Ameghino F. 1889. Contribución al conocimiento de los mamíferos fósiles de la República Argentina. Actas de la Academia Nacional de Ciencias de Córdoba, Parte III: 1486.

Bravard A. 1858. Monografía de los terrenos terciarios del Paraná. Anales del Museo Nacional de Historia Natural de Buenos Aires 3: 45-94.

Cabrera A. 1926. Cetáceos fósiles del Museo de La Plata. Revista del Museo de La Plata 28: 363-411.

Caviglia SE \& RE Jorge. 1980. Preaulophyseter gualichensis gen. et sp. nov. (Cetacea: Physeteridae), en el Terciario marino de Río Negro, República Argentina. Segundo Congreso Argentino de Paleontología y Bioestratigrafía y Congreso Latinoamericano de Paleontología 1, Actas 2: 363-370.
Cozzuol M. 1996. The record of the aquatic mammals in southern South America. Münchner Geowissenchaftliche Abhandlungen 30: 321-342.

De Muizon C. 1988. Les relations phylogénétiques des Delphinida (Cetacea, Mammalia). Annales de Paléontologie 74 (4): 159-257.

De Muizon C. 1991. A new Ziphiidae (Cetacea) from the Early Miocene of Washington State (USA) and phylogenetic analysis of major groups of odontocetes. Bulletin du Muséum National d'Histoire Naturelle 12 (34): 279-326.

De Muizon C, DP Domning \& DR Ketten. 2002. Odobenocetops peruvianus, the walrus-convergent delphinoid (Mammalia: Cetacea) from the Early Pliocene of Peru. En: Emry RJ (ed), Cenozoic mammals of land and sea: Tributes to the career of Clayton E. Ray. Smithsonian Contributions to Paleobiology 93: 223-261.

Gondar D. 1966. Cetacea. En: Borrello AV (ed). Paleontografía Bonaerense, Fascículo 4: 139-145. Comisión de Investigaciones Científicas, Provincia de Buenos Aires.

Gondar D. 1975. La presencia de cetáceos Physeteridae en el Terciario Superior ("Rionegrense”) de La Provincia de Río Negro. Actas del Primer Congreso Argentino de Paleontología y Bioestratigrafía 2: 349-356.

Kasuya T. 1973. Systematic consideration of recent toothed whale based on the morphology of tympano-periotic bone. Scientific Reports of the Whales Research Institute, Tokyo 25:1-103.

Kellogg R. 1944. Fossil cetaceans from the Florida Tertiary. Bulletin of the Museum of Comparative Zoology 94 (9): 433-71.

Luo Z. 1998. Homology and transformation of cetacean ectotympanic structures. En: Thewissen J (ed). The emergence of whales, pp. 269-301. Plenum Press, Nueva York.

Morgan GS. 1994. Miocene and Pliocene marine mammal faunas from the Bone Valley Formation of Central Florida. Proceedings of San Diego Society of Natural History 29: 239-268.

Sanders AE \& LG Barnes. 2002. Paleontology of the Late Oligocene Ashley and Chandler Bridge Formations of South Carolina, 3: Eomysticetidae, a new family of primitive mysticetes (Mammalia: Cetacea). Smithsonian Contributions to Paleobiology 93:313-356. 